\title{
Étude du comportement non-linéaire d'une colle dans un assemblage
}

\author{
Jean-Yves Cognard ${ }^{1, a}$, Laurent Sohier ${ }^{2}$, Peter Davies $^{3}$ et Bernard Gineste $^{2}$ \\ 1 Laboratoire de Mécanique des Structures Navales, ENSIETA, 2 rue F. Verny, 29806 Brest Cedex 09, France \\ ${ }^{2}$ Laboratoire d'Ingénierie Mécanique et Électrique, Université de Bretagne Occidentale, 29285 Brest Cedex, France \\ 3 Service Matériaux et Structures, IFREMER Centre de Brest, 29280 Plouzané, France
}

Reçu le 17 décembre 2004, accepté le 21 novembre 2005

\begin{abstract}
Résumé - L'objectif de cette étude est de définir à terme un outil fiable de dimensionnement d'assemblages collés pour favoriser l'utilisation de cette technique d'assemblage, en particulier, dans le domaine des structures marines et sous-marines. Un dispositif de type «Arcan », adapté pour l'étude du comportement d'un assemblage "métal-métal », a été développé pour se concentrer sur l'analyse du comportement de la colle en films minces. Pour analyser la cinématique de la déformation du joint de colle nous avons utilisé un système de mesure sans contact par corrélation d'images. Ainsi différentes caractéristiques du comportement non-linéaire de la colle ont été observées. Les premiers résultats obtenus pour les collages mixtes (acier, aluminium, composite) montrent des comportements similaires de la colle pour la procédure proposée. De plus, des contrôles de qualité par DSC, et des études de cuisson par DMA sont réalisées pour vérifier la conformité de la procédure de collage.
\end{abstract}

Mots clés : Assemblages collés / modélisation / essais / comportement non-linéaire / composites

Abstract - Analysis of the non-linear behaviour of an adhesive in an assembly. The objective of this study is to define a reliable tool for dimensioning of adhesively bonded assemblies, particularly for marine and underwater applications. A modified "Arcan" fixture, suited for the study of the behavior of bonded metal-metal assemblies, was developed in order to focus on the analysis of the behavior of the adhesive in thin films. To analyze the kinematics of the deformation of the adhesive joint non-contact extensometry techniques were employed. Thus various characteristics of the non-linear behavior of the adhesive were observed. The first results obtained for mixed assemblies (steel, aluminium, composite substrates) show similar behaviour of the adhesive using the proposed procedure. Furthermore, quality controls by DSC, and studies of curing by DMA have been performed to verify the bonding procedure.

Key words: Adhesive bonding / model / tests / non linear behaviour / composite

\section{Introduction}

Cette étude se situe dans le cadre de la recherche de gains de performance de structures composites par l'utilisation d'assemblages collés, les applications concernent plus particulièrement le domaine des structures marines et sous-marines. Elle est menée dans le cadre d'un Programme de Recherche d'Intérêt Régional (Région Bretagne) réalisé en partenariat entre IFREMER, L'ENSIETA, l'UBO, l'ENS de Cachan et deux entreprises du secteur du nautisme hautes performances (une unité de réalisation : MULTIPLAST et un

\footnotetext{
a Auteur correspondant: jean.yves.cognard@ensieta.fr
}

bureau de calculs : HDS). Les assemblages collés offrent de nombreux avantages pour la réalisation de structures marines, mais un manque de confiance des bureaux d'études limite aujourd'hui l'utilisation de cette technologie [1-4]. Les applications industrielles visées, en particulier les assemblages collés mixtes (collage métal composite), sont caractérisées par l'utilisation de joints de colle relativement épais, de l'ordre de $0,5 \mathrm{~mm}$. De plus, ces joints peuvent avoir une épaisseur non-constante du fait de la géométrie et des grandes dimensions des pièces à assembler.

L'objectif est de définir à terme un outil fiable de dimensionnement d'assemblages collés de structures composites, ainsi que des méthodologies de contrôle qualité 
pour bénéficier de gains de performances. Notre démarche consiste à définir un moyen d'essai permettant de caractériser la colle en film mince, puis à quantifier l'influence des paramètres de construction des structures de type naval pour intégrer l'ensemble de ces développements dans un outil de dimensionnement.

Il existe un essai normalisé qui permet de déterminer la courbe contrainte-déformation : c'est un essai de cisaillement avec substrats épais [5]; mais, cet essai ne permet d'étudier que le cisaillement. De plus, pour la colle qui nous intéresse (Redux 420), le fabricant ne donne que très peu de caractéristiques mécaniques [6]. Ces données ne sont donc pas suffisantes pour le dimensionnement de structures collées. Ainsi nous proposons de développer une stratégie de caractérisation du comportement nonlinéaire de la colle; les conditions de fabrication des chantiers nautiques (collage de pièces de grandes dimensions, préparation simple des surfaces et post cuisson à une faible température) sont utilisées pour la mise en œuvre des essais.

Dans une première étude, un dispositif de type « Arcan », adapté pour l'étude du comportement d'un assemblage «métal-métal », a été développé pour se concentrer sur l'analyse du comportement de la colle en films minces et un système de «becs » a été proposé pour supprimer les effets de bords $[7,8]$. L'enveloppe de rupture en traction-compression/cisaillement obtenue pour la colle Redux 420 montre que la compression augmente fortement la résistance au cisaillement.

Cet article présente la stratégie utilisée et les résultats de l'analyse du comportement non-linéaire de la colle sous différentes sollicitations (monotone, cyclique, complexe... ). Pour analyser la cinématique de la déformation du joint de colle nous avons choisi d'utiliser un système de mesure sans contact par corrélation d'images. L'étude directe des déplacements dans la colle est difficile du fait de sa géométrie non-plane en périphérie, de sa très faible épaisseur et de la présence des becs. Ainsi nous analysons, par une technique d'optimisation, l'évolution en fonction du temps des déplacements des deux substrats. Les différents essais réalisés montrent les différentes phases du comportement de type visco-élasto-plastique de la colle : une phase associée à une faible déformation anélastique et une phase caractérisée par une déformation viscoplastique assez importante, en particulier pour les sollicitations de cisaillement.

Pour étudier l'influence de la procédure de collage des contrôles de qualité par DSC, et des études de cuisson par DMA sont aussi réalisées. Pour poursuivre l'étude, une adaptation du montage d'essai de type « Arcan » est à l'étude pour étudier le comportement d'assemblages collés composite-métal et composite-composite.

\section{Conception du dispositif expérimental}

\subsection{Problème à résoudre}

Pour caractériser le comportement de la colle il faut utiliser un dispositif où le joint de colle étudié a une

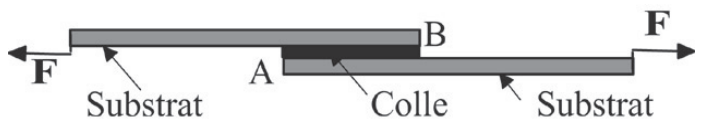

a) joint à simple recouvrement

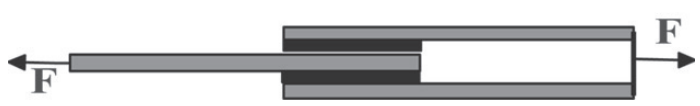

b) joint à double recouvrement

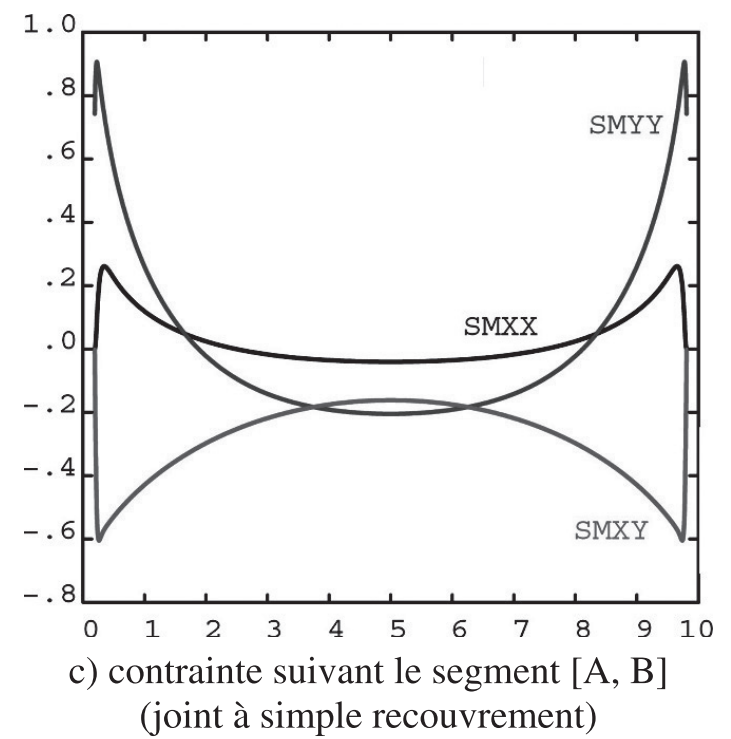

Fig. 1. Principes de quelques dispositifs expérimentaux.

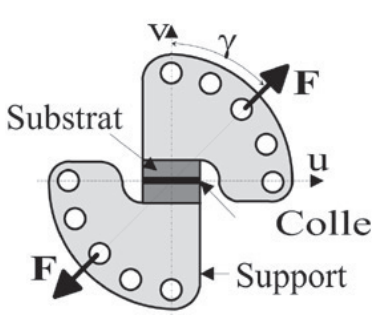

a) montage «Arcan»

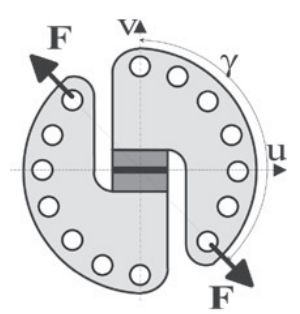

b) montage modifié
Fig. 2. Montages de type «Arcan ».

géométrie similaire à celle utilisée lors de la réalisation d'assemblages, c'est-à-dire une épaisseur de l'ordre de quelques dixièmes de millimètres [9]. Les éprouvettes de type simple ou double recouvrement (Figs. 1a, b), sont d'une mise en ouvre facile mais génèrent des forts gradients de contrainte (points A et B, Fig. 1c) [10]. De plus, avec ce type de dispositif il n'est pas facile d'étudier l'influence de la contrainte normale sur la résistance au cisaillement de la colle.

Il existe un essai normalisé qui permet de déterminer la courbe contrainte-déformation : c'est un essai de cisaillement avec substrats épais [5]; mais, cet essai ne permet d'étudier que le cisaillement. Ainsi pour solliciter le joint de colle, dans le plan « contrainte normale contrainte tangentielle $»$ nous proposons d'utiliser un dispositif de type «Arcan» [11] (Fig. 2a), permettant de solliciter aussi la colle en compression (Fig. 2b). 

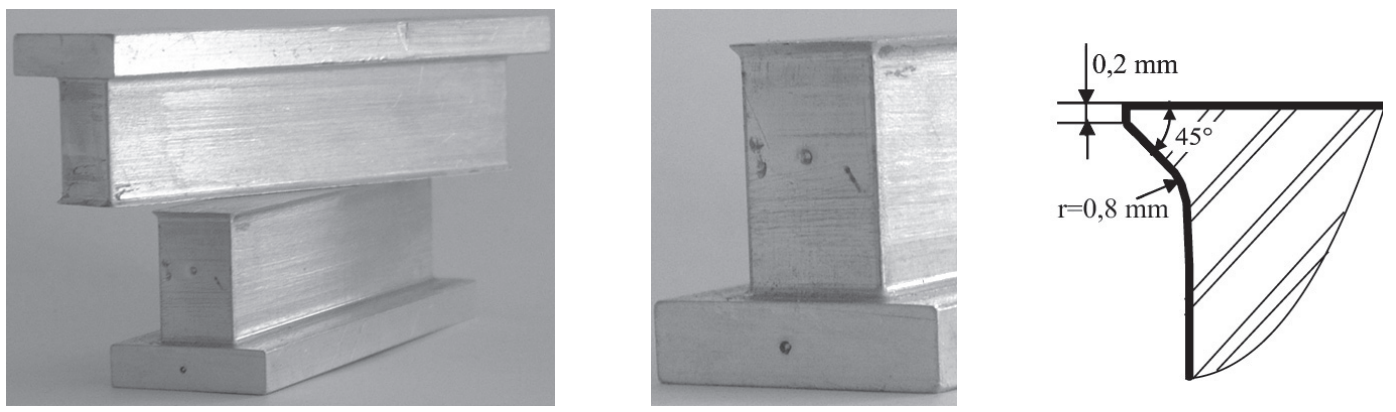

Fig. 3. Géométrie des substrats avec le bec.

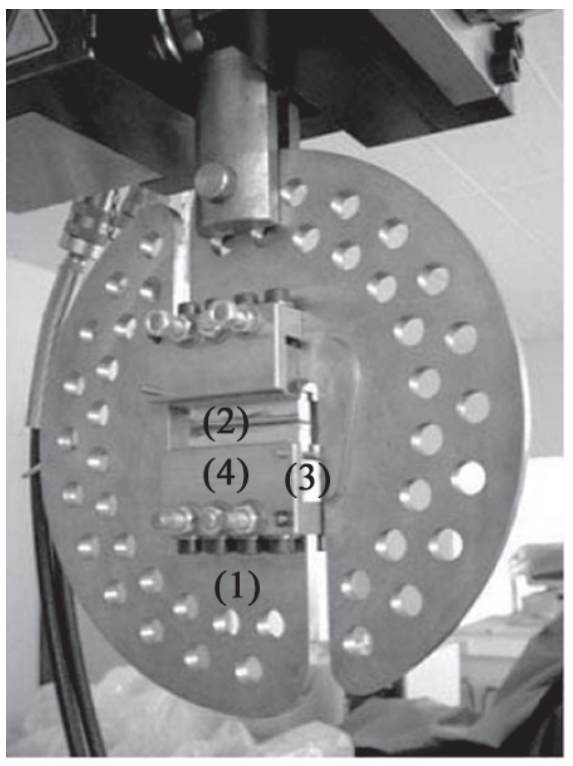

a) montage « Arcan »

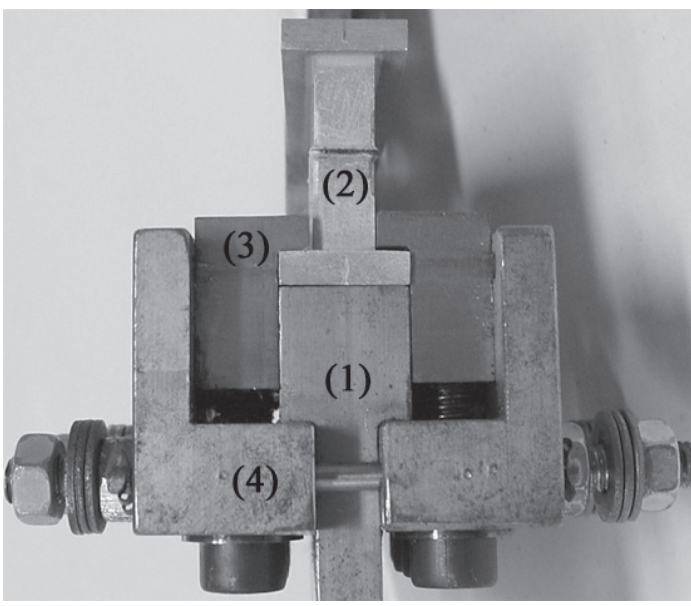

(1) support du montage «Arcan »

(2) éprouvette collée

(3) mors de serrage

(4) support du mors de serrage

b) fixation de l'éprouvette

Fig. 4. Présentation du montage «Arcan».

\subsection{Conception du dispositif expérimental}

Deux points importants ont été étudiés lors de la conception de ce montage :

- obtenir un champ de contrainte le plus uniforme possible dans le plan moyen du joint de colle et maximal au centre pour limiter les effets des défauts,

- concevoir un système d'accrochage de l'éprouvette (substrat) sur le support du montage «Arcan» qui ne perturbe pas le champ de contrainte dans la colle.

Des études en élasticité linéaire, pour des structures réalisées en bi-matériaux, montrent que l'utilisation d'un bec permet d'éliminer la contribution des singularités dues aux effets de bord [11,12]. Le système d'accrochage du substrat sur le support peut générer une précharge non uniforme dans le joint de colle et ainsi rendre l'essai difficilement exploitable. Différentes simulations ont permis de définir, un dispositif d'accrochage adéquat [8]. Pour refléter la réalité, les modélisations éléments finis utilisées prennent en compte les contacts avec frottement et les procédures de montage. Pour conserver la simplicité associée au montage « Arcan », et l'utilisation d'une machine de traction classique, une éprouvette à section rectangulaire (section de $70 \times 10 \mathrm{~mm}$ ) a été proposée en prenant en compte les problèmes liés à l'usinage (Fig. 3). Le bec est usiné avec un angle de $\alpha=45^{\circ}$ et un rayon de raccordement de $0,8 \mathrm{~mm}$. La réalisation d'un bec avec un angle vif pose des problèmes de fabrication et d'utilisation. Des simulations ont montré qu'une surépaisseur de l'ordre de 0,2 mm sur les becs ne perturbait pas le champ de contrainte dans la colle.

La figure 4a présente le montage «Arcan » utilisé et la figure 4b montre le montage de l'éprouvette collée dans le dispositif « Arcan ». Les mors de serrage participent au positionnement de l'éprouvette collée par rapport au support du montage «Arcan » et à son maintien par serrage.

\subsection{Analyse des contraintes dans le plan moyen de la colle}

La propriété de symétrie par rapport au plan de chargement $(O, X, Y), O$ étant le centre de l'éprouvette collée et la propriété de type «anti-symétrie » du chargement permettent de réduire cette simulation tridimensionnelle 


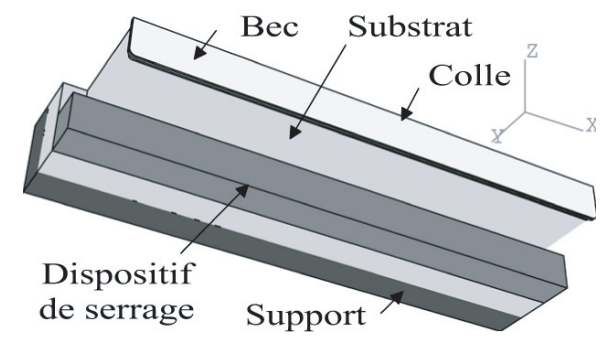

a) modèle utilisé

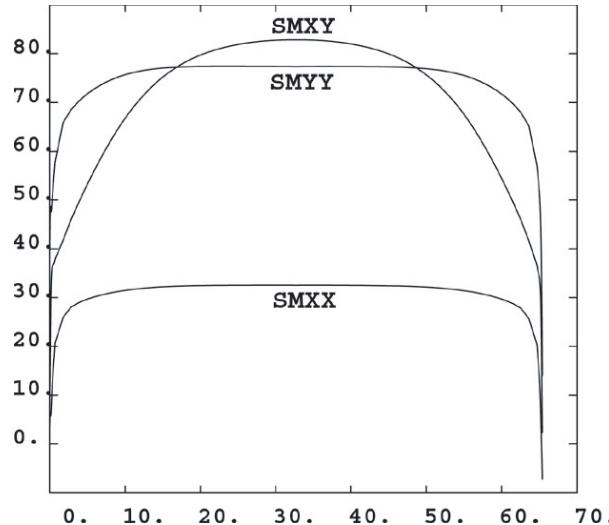

b) contrainte suivant la droite $(\mathrm{O}, \mathrm{X})$
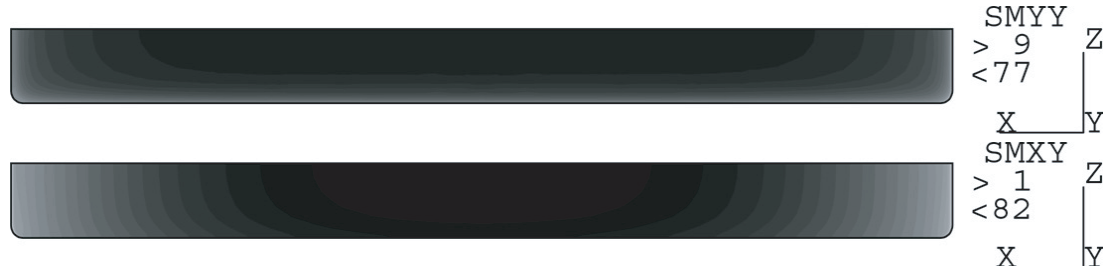

0 .

12 .

32.

43 .

53.

63.

74.

c) contrainte dans le plan moyen de la colle

Fig. 5. Répartition des contraintes dans le plan moyen de la colle pour le dispositif proposé.

au quart du dispositif en appliquant les conditions aux limites adéquates. Après la simulation du serrage de l'éprouvette, une sollicitation de traction-cisaillement est imposée sur la face supérieure du support. La recherche de résultats numériques précis conduit à des modèles de grande taille (plus de $500000 \mathrm{ddl}$ ) [13,14]; ces simulations ont été réalisées avec CAST3M [15]. La figure 5 présente la répartition des contraintes dans le plan moyen de la colle; elle montre que les contraintes sont maximales au centre de l'éprouvette et qu'elles s'annulent sur la périphérie. L'éprouvette proposée respecte donc le cahier des charges imposé, mais l'identification des paramètres du comportement de la colle nécessitera une technique d'identification inverse. Sous les hypothèses du calcul, les valeurs maximales des composantes de la contrainte sont obtenues à partir des résultats éléments finis et de la contrainte moyenne obtenue à partir des efforts mesurés lors de l'essai et de la section du plan de colle $\left(S_{\mathrm{c}}\right)$ :

$$
\begin{array}{lll}
\sigma_{y y \text { maxi }}=1.12 \sigma_{y y \text { moyen }} & \text { avec } & \sigma_{y y \text { moyen }}=F_{y} / S_{\mathrm{c}} \\
\sigma_{x y \text { maxi }}=1.29 \sigma_{x y \text { moyen }} & \text { avec } & \sigma_{x y \text { moyen }}=F_{x} / S_{\mathrm{c}}
\end{array}
$$

Ces valeurs sont importantes pour l'identification des propriétés de la colle.

\subsection{Procédure expérimentale}

Les dimensions du joint de colle étant relativement faibles $(70 \times 10 \mathrm{~mm})$, il faut éviter au maximum les sollicitations parasites, pouvant entraîner une modification des contraintes dans le joint de colle [8]. Les points importants à contrôler sont :

- la qualité géométrique des différentes pièces,

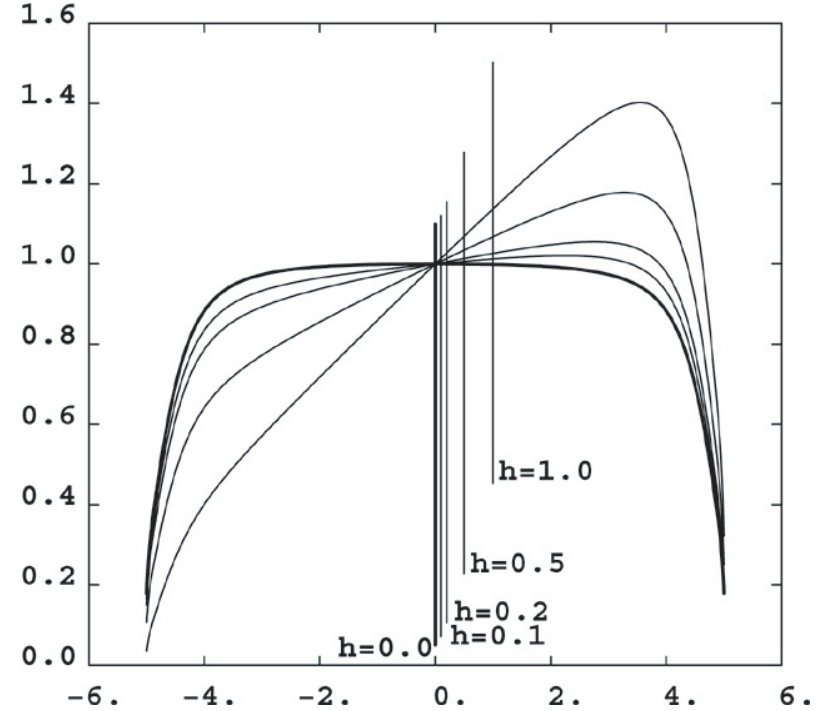

Fig. 6. Influence d'un défaut de positionnement sur la répartition des contraintes.

- le positionnement relatif des deux substrats lors du collage,

- le positionnement de l'éprouvette dans le support du montage « Arcan »,

- la liaison entre le montage «Arcan » et la machine de traction (position des rotules).

Pour évaluer les défauts maximums admissibles lors de mise en œuvre de l'essai, différentes simulations ont été réalisées à partir d'une modélisation $2 \mathrm{D}$ dans le plan $(O, Y, Z)$. La figure 6 présente l'influence d'une excentration du chargement par rapport au plan moyen 


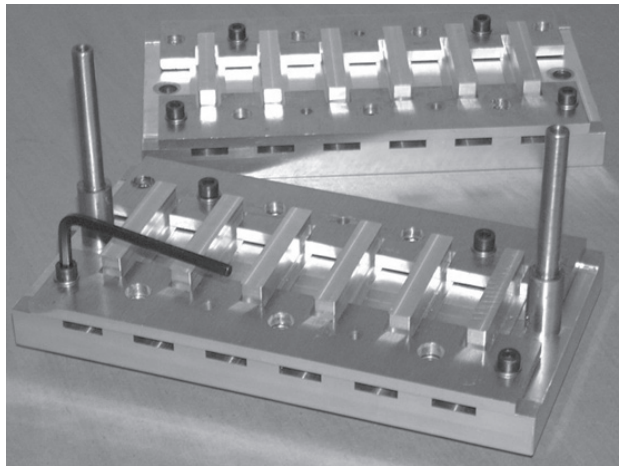

a) montage de collage

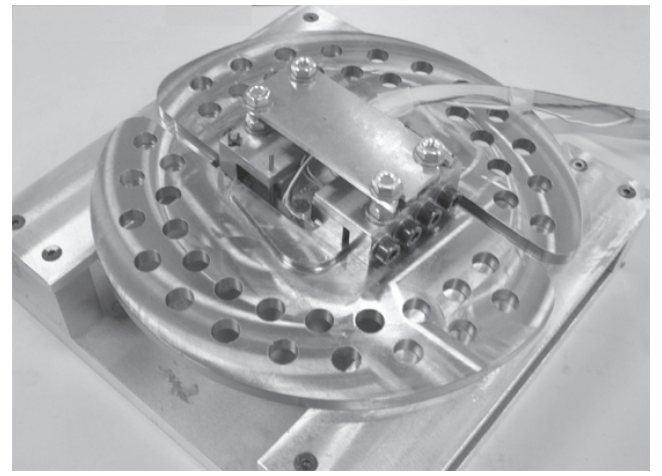

b) dispositif de montage des éprouvettes

Fig. 7. Montage de collage et dispositif de montage des éprouvettes.

de l'éprouvette (paramétrée par la distance $h$ ) sur la répartition des contraintes normales dans la colle. Il est important de noter la sensibilité de ce paramètre : d'après ces calculs, pour limiter à $5 \%$ la modification des contraintes normales maximales il faut une excentration du chargement inférieure à $h=0,2 \mathrm{~mm}$. Ces calculs confirment la nécessité de vérifier les différents points cités précédemment pour définir la précision des essais.

Le respect du positionnement des substrats est obtenu par l'utilisation d'un montage de collage (Fig. 7a). Un appairage des substrats deux à deux permet de garantir une bonne maîtrise de l'épaisseur et de la géométrie des collages. Pour assurer un bon positionnement relatif des substrats dans le montage «Arcan » un dispositif de montage est utilisé (Fig. 7b). De plus, lors de la fixation du montage «Arcan» sur la machine de traction, des plaques de protection évitent les sollicitations du joint de colle. Des éprouvettes instrumentées (Fig. 7b) ont été utilisées pour valider les procédures proposées et ainsi limiter au maximum les chargements parasites.

\section{Analyse de la cinématique de la déformation du joint de colle}

Les résultats des analyses du comportement de la colle, présentés dans cette partie, sont obtenus pour des substrats en aluminium et une épaisseur du joint de colle de $0.4 \pm 0.02 \mathrm{~mm}$.

\subsection{Système de mesure}

Pour analyser la cinématique de la déformation du joint de colle nous avons choisi d'utiliser un système de mesure sans contact par corrélation d'images [16]. Ce système permet, dans un premier temps, d'observer le comportement de la colle puis d'analyser plus finement par un post traitement les caractéristiques géométriques du joint de colle au cours du chargement. L'analyse des images a montré un bon comportement du montage au cours de l'essai. Nous avons observé que le plan moyen

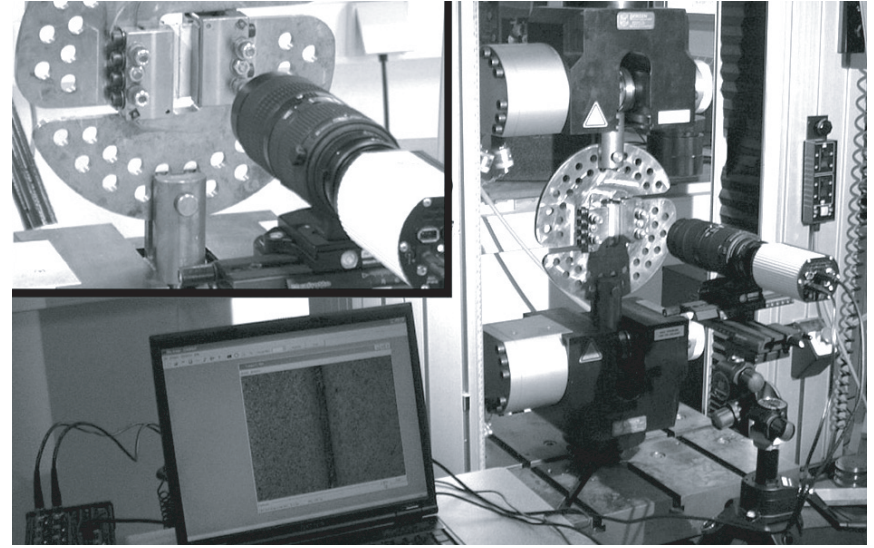

Fig. 8. Présentation du dispositif expérimental.

du joint de colle se déplace toujours parallèlement à luimême au cours de l'essai; ceci confirme que le chargement du joint de colle en terme de sollicitation normale et tangentielle reste constant au cours du temps pour un essai donné. L'étude directe, par analyse d'images, des déplacements et déformations dans le joint de colle est difficile du fait de sa géométrie non-plane en périphérie de l'éprouvette, de sa très faible épaisseur (de l'ordre de $0,4 \mathrm{~mm}$ ) et de la présence des becs. Ainsi nous étudions les déplacements des deux substrats; de plus, pour obtenir une bonne précision sur les déplacements par analyse d'images, une zone de l'éprouvette d'environ $1 \mathrm{~cm} \times 1 \mathrm{~cm}$ est analysée avec des images de 1280 pixels $\times 1024$ pixels.

La figure 8 présente le dispositif expérimental utilisé dans le cas d'un essai de cisaillement de la colle $\left(90^{\circ}\right)$. Pour cet essai la vitesse de déplacement de la traverse de la machine de traction est imposée à $0,5 \mathrm{~mm} \cdot \mathrm{min}^{-1}$ et une image est prise toutes les secondes. Les figures $9 \mathrm{a}, \mathrm{b}$ présentent les images à l'instant initial et juste avant rupture. Sur cette dernière image, il est possible de visualiser les déformations de la colle; en effet, des «stries » à $45^{\circ}$ apparaissent de façon homogène dès le début du comportement non-linéaire de la colle et le mouvement relatif des deux substrats est directement perceptible sur les images. Par contre, pour des faibles efforts le mouvement relatif des deux substrats n'est pas directement observable. 


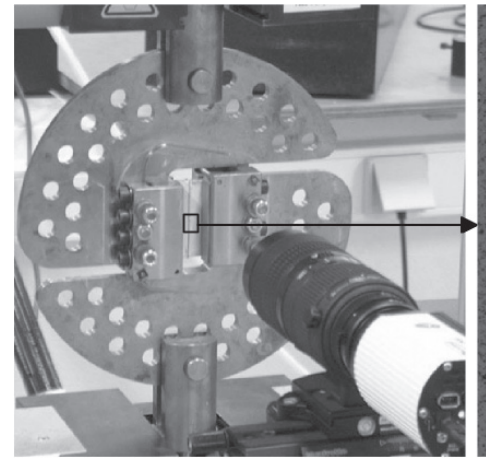

(a) image initiale et zones étudiées

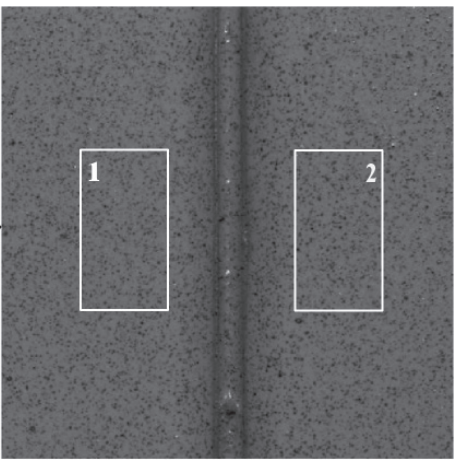

(b) juste avant rupture (zoom)

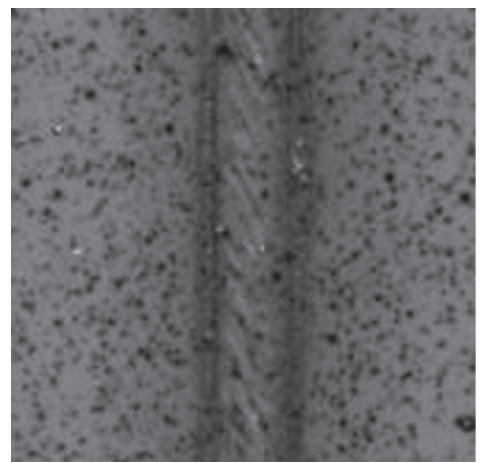

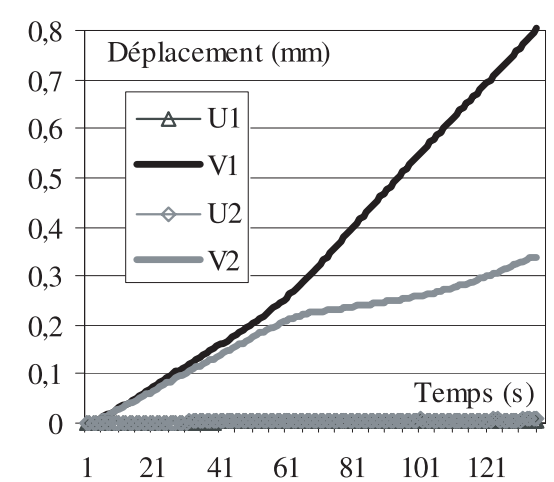

(c) déplacement mesuré pour les deux substrats

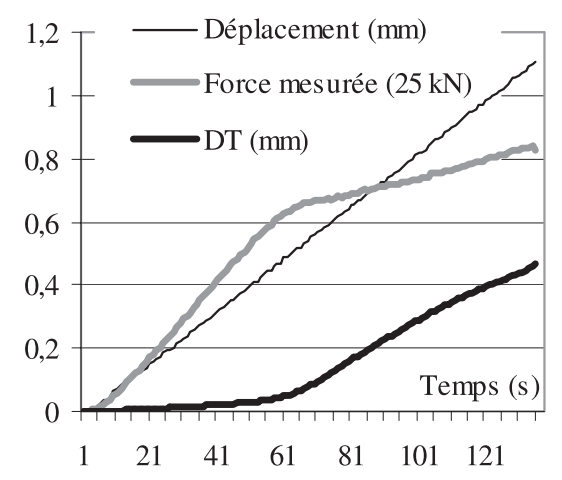

(d) évolution en temps de différentes quantités

Fig. 9. Analyse de la cinématique de la déformation du joint de colle (cisaillement).

\subsection{Analyse des déplacements dans le joint de colle}

Les deux zones d'étude sont prises de façon symétrique par rapport au joint de colle pour permettre une analyse du déplacement des deux substrats (Fig. 9c). Ensuite après obtention du champ de déplacement dans les deux zones d'étude par corrélation d'images, une technique d'optimisation est utilisée pour obtenir le meilleur champ de déplacement des substrats (zone d'étude). Cette technique a été utilisée avec une modélisation de mouvement de corps rigide pour les substrats ou une modélisation prenant en compte les déformations des substrats associées aux sollicitations de traction et cisaillement. Les sollicitations mécaniques des substrats en aluminium étant relativement faibles, les deux approches donnent des résultats similaires sur les déplacements relatifs des deux extrémités du joint de colle.

Par exemple, sous l'hypothèse de comportement rigide des substrats, le champ de déplacement $\left(U_{\mathrm{Ri}}, V_{\mathrm{Ri}}\right)$ est recherché, en chaque point $M_{i}\left(X_{i}, Y_{i}\right)$ d'une zone, sous la forme suivante :

$$
\begin{aligned}
& U_{\mathrm{Ri}}=U_{0}-\Omega Y_{i} \\
& V_{\mathrm{Ri}}=V_{0}+\Omega X_{i}
\end{aligned}
$$

où $U_{0}$ et $V_{0}$ sont les composantes du déplacement de l'origine et $\Omega$ représente la rotation. Les trois inconnues $\left\{U_{0}\right.$, $\left.V_{0}, \Omega\right\}$ sont obtenues par la minimisation d'une distance entre les valeurs modélisées des déplacements et les valeurs mesurées pour les points $M_{i}\left(U_{M i}, V_{M i}\right)$. Les valeurs des déplacements mesurés sont connus pour les $N$ pixels de la zone d'étude. En résolvant le problème de minimisation suivant :

Minimisation de $\Sigma_{(i=1, N)}\left\{\left(U_{\mathrm{Ri}}-U_{M i}\right)^{2}+\left(V_{\mathrm{Ri}}-V_{M i}\right)^{2}\right\}$ les inconnues sont les solutions d'un problème linéaire à trois équations pour chaque instant :

$$
\begin{array}{rcc}
{\left[\begin{array}{ccc}
N & 0 & -\Sigma\left(Y_{i}\right) \\
0 & N & \Sigma\left(X_{i}\right) \\
-\Sigma\left(Y_{i}\right) & \Sigma\left(X_{i}\right) & \Sigma\left(X_{i}\right)^{2}+\Sigma\left(Y_{i}\right)^{2}
\end{array}\right]\left[\begin{array}{c}
U_{0} \\
V_{0} \\
\Omega
\end{array}\right]=} \\
\\
{\left[\begin{array}{c}
\Sigma\left(U_{M i}\right) \\
\Sigma\left(V_{M i}\right) \\
\Sigma\left(X_{i} V_{M i}\right)-\Sigma\left(Y_{i} V_{M i}\right)
\end{array}\right]}
\end{array}
$$

Ainsi une représentation de l'évolution en temps des déplacements des substrats est obtenue; en fait, pour chaque image nous obtenons une valeur des déplacements des substrats.

\subsection{Analyse d'un chargement monotone du joint de colle}

Nous notons respectivement DN et DT les déplacements relatifs des deux extrémités du joint de colle respectivement dans les directions normale et tangentielle au plan moyen du joint de colle. La figure $9 \mathrm{~d}$ présente l'évolution en fonction du temps des déplacements horizontaux $\left(U_{i}\right)$ et verticaux $\left(V_{i}\right)$ des 


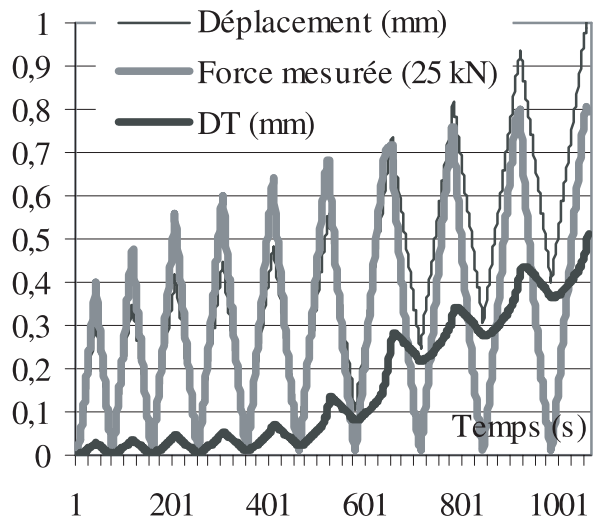

(a) évolution en temps de différentes quantités

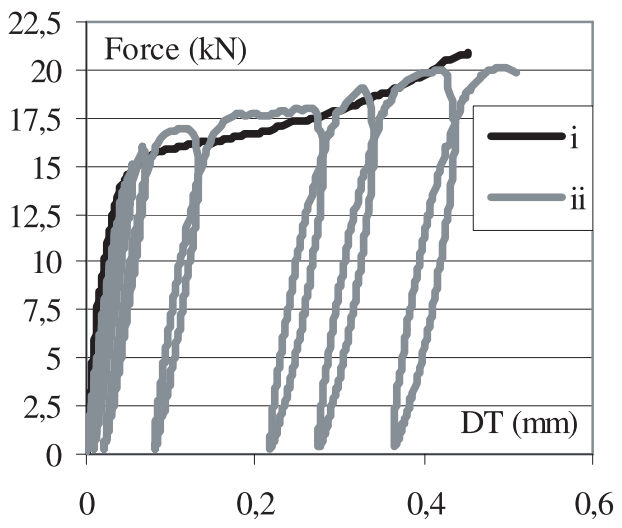

(i) chargement monotone

(ii) chargement cyclique

(b) diagramme force-déplacement

Fig. 10. Comportement en cisaillement sous chargement cyclique.

centres des deux zones analysées. Il est important de noter que les déplacements horizontaux sont quasiment nuls, ce qui souligne le bon comportement du dispositif expérimental. Enfin, la figure 10a donne l'évolution en fonction du temps du déplacement imposé à la traverse de la machine de traction, la force exercée par la machine de traction sur l'éprouvette et le déplacement tangentiel DT du joint de colle. Le déplacement imposé par la traverse de la machine de traction ne correspond pas directement au déplacement de la colle; en effet, il y a des déformations du montage et celui-ci est relié à la machine de traction par l'intermédiaire de liaisons ponctuelles pour assurer l'isostaticité. Nous pouvons remarquer que pour de faibles efforts, le déplacement relatif des deux extrémités de la colle est faible.

Pour cet essai, sous chargement monotone, nous pouvons constater deux zones dans le comportement de la colle :

- Une zone que nous qualifierons de «linéaire» caractérisée par une relation linéaire entre la force appliquée et le déplacement DT des deux extrémités du joint de colle; de plus cette zone est associée à une faible évolution de la déformation;

- Une zone à comportement non-linéaire caractérisée par une évolution importante de la déformation $\left(\mathrm{DT}_{\operatorname{maxi}}=0,45 \mathrm{~mm}\right.$ pour une épaisseur du joint de $0,5 \mathrm{~mm})$.

Une analyse des incertitudes de la procédure utilisée est nécessaire pour qualifier les résultats obtenus. La valeur minimale de déplacement détectable par les stratégies de corrélation d'images utilisant des techniques de résolutions multi-échelle est inférieure à 0,03 pixel, pour des images codées sur 8 bits [17]. Ainsi, pour le facteur d'échelle utilisé qui correspond à 100 pixels par mm, l'ordre de grandeur du déplacement minimal mesurable est donc de $3 \times 10^{-4} \mathrm{~mm}$. Comme le résultat que nous obtenons est la différence de deux déplacements mesurés par analyse d'images associé au déplacement relatif des substrats, l'incertitude de mesure est plus grande que cette valeur; de plus, il faut prendre en compte les effets de toute la chaîne d'acquisition et d'analyse. L'analyse des résultats obtenus montre que nous avons une incertitude inférieure à $\pm 0,15$ pixel $( \pm 0,0015 \mathrm{~mm})$ pour un déplacement relatif inférieur à 3 pixels $(0,03 \mathrm{~mm})$ c'est-à-dire pour le début de la zone de comportement « linéaire ». Par contre, dans la zone de comportement non-linéaire, nous avons une incertitude inférieure à $\pm 0,1$ pixel $( \pm 0,001 \mathrm{~mm})$ pour des déplacements relatifs compris entre 5 et 50 pixels (entre $0,05 \mathrm{~mm}$ et $0,5 \mathrm{~mm}$ ). Il est important de noter que l'incertitude de mesure diminue avec la valeur du déplacement mesuré; ainsi, l'erreur relative diminue fortement avec la valeur du déplacement. Pour les faibles efforts la liaison ponctuelle, utilisée pour assurer l'isostaticité du montage, peut générer des petites perturbations liées au frottement qui s'ajoutent aux dispersions de mesure.

Une préparation très soignée de l'essai (qualité du mouchetis, éclairage, ...) conduit à des incertitudes plus faibles. La démarche utilisée nécessite donc des déplacements relatifs des substrats supérieurs à 1 pixel pour obtenir des résultats de bonne qualité. La qualité des résultats peut être améliorée par l'utilisation d'un facteur d'agrandissement plus grand (zone d'analyse plus petite ou caméra plus précise) et par l'utilisation d'images codées sur plus de 8 bits (caméra plus précise).

\section{4 Étude du comportement non-linéaire du joint de colle}

La figure 10 présente les résultats d'un essai en cisaillement sous chargement cyclique avec une valeur croissante de la charge. Ces résultats montrent un comportement élasto-visco-plastique de la colle. La figure 11 présente le comportement de la colle pour des sollicitations de 


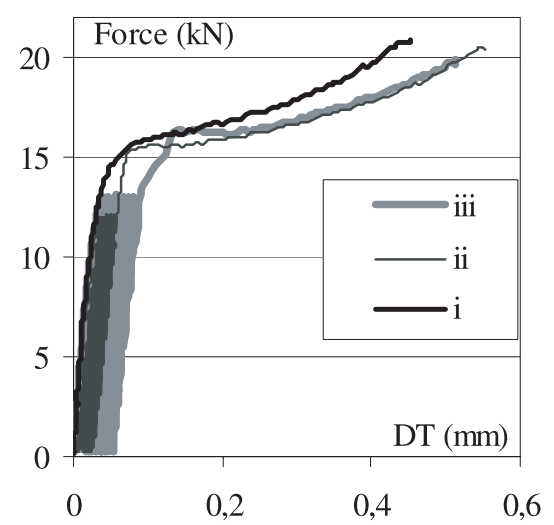

(i) chargement monotone

(ii) 20 cycles à $12 \mathrm{kN}$ puis chargement monotone

(iii) 20 cycles à $13 \mathrm{kN}$ puis chargement monotone

(a) cisaillement $\left(90^{\circ}\right)$

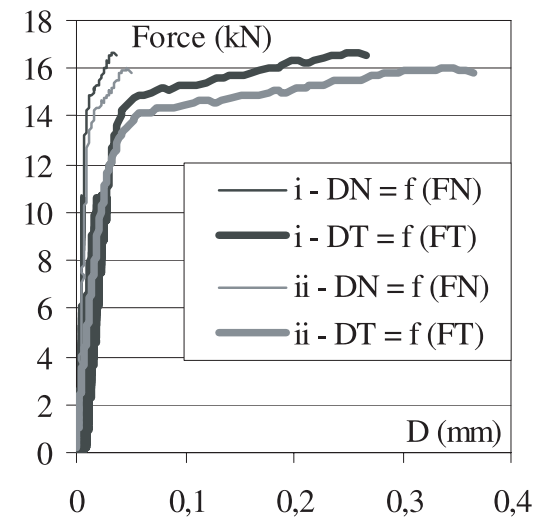

(i) chargement monotone

(ii) 20 cycles à $15 \mathrm{kN}$ puis chargement monotone

(b) traction-cisaillement $\left(45^{\circ}\right)$

Fig. 11. Comportement sous chargement cyclique dans la zone de comportement « linéaire ».

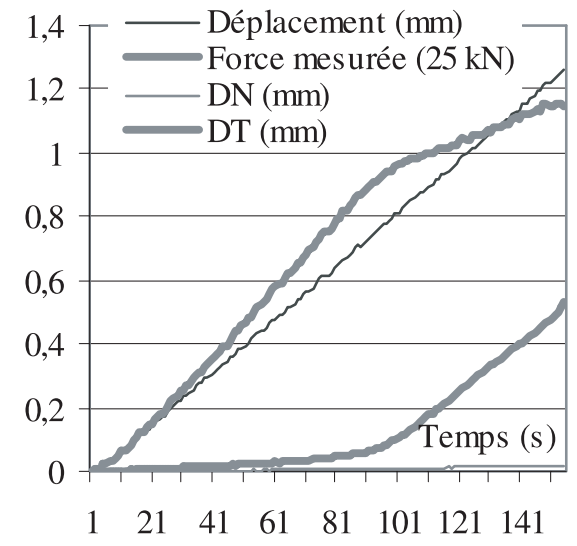

(a) chargement monotone

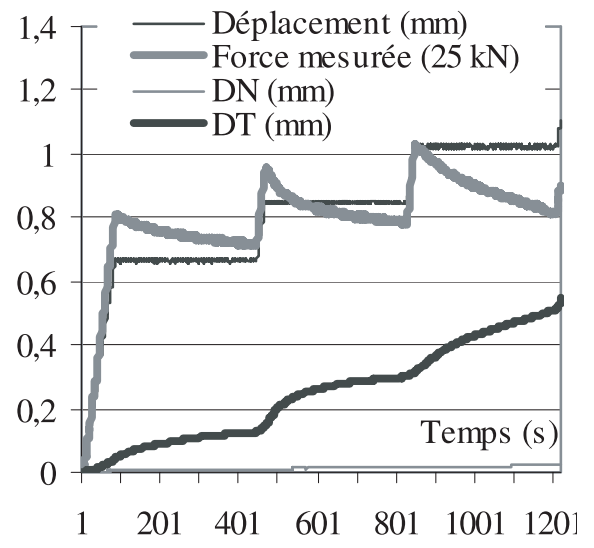

(b) chargement complexe

Fig. 12. Comportement pour des chargements de compression-cisaillement $\left(135^{\circ}\right)$.

cisaillement et de traction-cisaillement sous chargement monotone et sous chargements cycliques (20 cycles) suivie d'un chargement monotone. FN et FT représentent respectivement les composantes de l'effort appliqué dans les directions normale et tangentielle au plan moyen du joint de colle. Ces résultats montrent qu'il y a de faibles déformations anélastiques pour des faibles niveaux de chargement, c'est-à-dire pour les sollicitations dans la zone de comportement « linéaire » observée pour un chargement monotone. La figure 12 illustre le comportement de la colle sous chargement de compression-cisaillement. Pour simplifier la représentation les valeurs absolues des composantes normales et tangentielles des déplacements et des efforts sont présentées. Pour des chargements de type « relaxation », obtenus en bloquant la traverse de la machine de traction, nous observons une augmentation de la déformation de la colle et une diminution de l'effort transmis par le joint de colle. Il est aussi important de noter que la composante normale (DN) du déplacement de la colle est très inférieure à la composante tangentielle (DT) (de l'ordre de $1 / 20$ en compression et $1 / 10$ en traction). Pour la sollicitation de traction le dispositif expérimental utilisé ne permet pas d'obtenir avec suffisamment de précision l'évolution de la cinématique de déformation de la colle : une autre technique de mesure doit donc être utilisée. Pour les autres sollicitations la combinaison des différents déplacements rend possible la mesure avec une bonne précision.

\section{Courbes enveloppes en contrainte}

La figure 13 donne, dans le plan contrainte normale - contrainte tangentielle, l'évolution des contraintes à rupture pour la colle Redux 420. Après assemblage, les éprouvettes sont laissées 12 heures à $20{ }^{\circ} \mathrm{C}$ puis maintenues à $50{ }^{\circ} \mathrm{C}$ pendant 4 heures. Ces résultats sont obtenus pour des substrats en aluminium, une épaisseur 


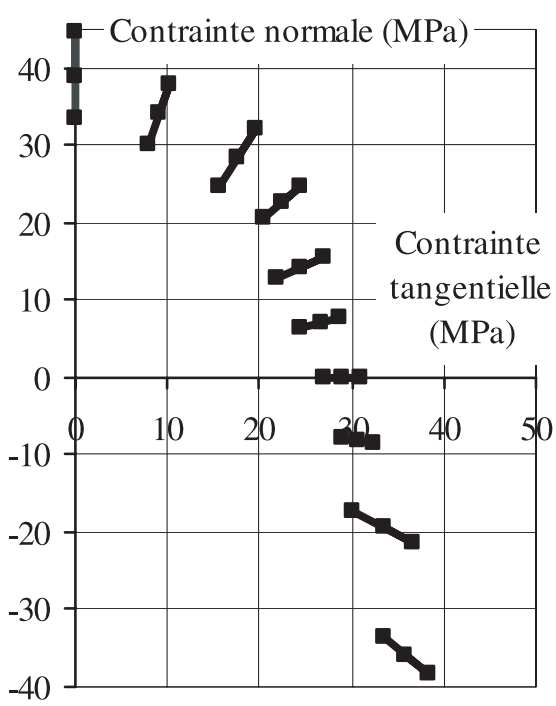

(a) enveloppe de rupture

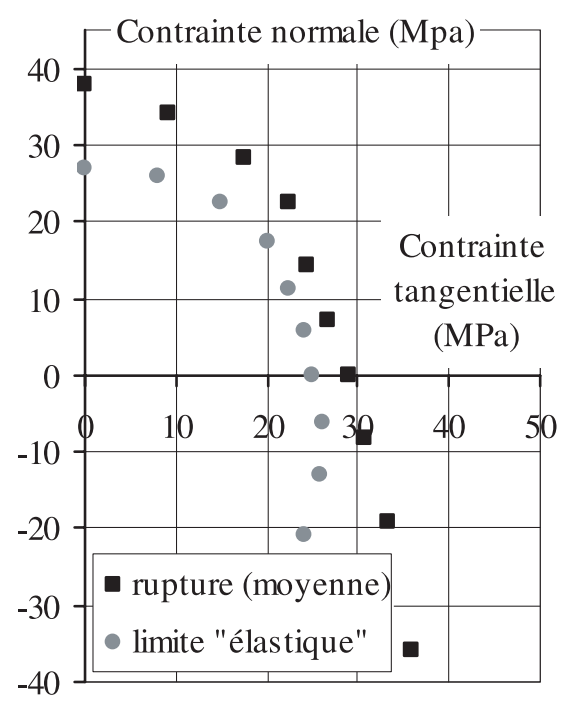

(b) enveloppe "élastique" et de rupture

Fig. 13. Comportement en traction/compression-cisaillement, colle Vantico Redux 420.

du joint de colle de $0.4 \pm 0.02 \mathrm{~mm}$ et une vitesse imposée $0.5 \mathrm{~mm} \cdot \mathrm{min}^{-1}$ pour la traverse de la machine de traction. Sous l'hypothèse d'un comportement de type élastoplastique avec écrouissage isotrope linéaire, la simulation d'un essai de cisaillement de la colle montre que l'on retrouve le comportement global observé lors des essais pour un chargement monotone et qu'après la phase transitoire, l'état de contrainte est uniforme dans la colle. Ainsi, proche de la rupture, nous pouvons supposer une répartition uniforme des contraintes dans le plan moyen de la colle (redistribution des contraintes associée au comportement plastique) ainsi la courbe enveloppe de rupture est tracée avec les contraintes moyennes. Il est important de noter que la résistance en traction est presque identique à celle obtenue en cisaillement. De plus, la compression augmente fortement la résistance au cisaillement, ce qui est très important pour les applications sousmarines. Par ailleurs, l'examen des faciès montre une rupture de type adhésive (rupture à l'interface colle-substrat) et cohésive (rupture dans le joint de colle) pour des sollicitations où la contrainte normale est dominante et de type adhésive mixte (rupture aux interfaces colle-substrat et dans la colle perpendiculairement au plan moyen) dans les autres cas. Pour une utilisation pratique de l'enveloppe de résistance en terme de contrainte sous chargements monotones, il semble utile de déterminer le domaine « linéaire »; celui-ci est tracé, figure $13 \mathrm{~b}$, avec les valeurs maximales des contraintes calculées sous l'hypothèse de comportement élastique (formule (1)).

Il est important de noter que les performances maximales sont obtenues avec de la colle réalisée juste après ouverture des pots. Comme certains essais sont réalisés avec de l'adhésif réalisé plusieurs jours après la première ouverture des pots, les dispersions obtenues sont donc supérieures à celle obtenue sur des chantiers nautiques qui utilisent des grandes quantités de colle.

\section{5 Étude du collage mixte}

Des essais ont été réalisés avec des substrats en acier et aluminium. Les résultats obtenus (Fig. 14) montrent que la nature des substrats a très peu d'influence sur l'analyse du comportement de la colle. Cependant il est important de noter qu'une préparation adéquate des surfaces doit être réalisée en fonction de la nature des matériaux.

Pour analyser le comportement de collage de composite, un test de validation a été réalisé. Une plaque de composite (trois plis d'un tissu équilibré $0^{\circ} / 90^{\circ}$ en carbone haute résistance de $193 \mathrm{~g} . \mathrm{m}^{-2}$ et résine époxyde SR1500) a été collée entre deux substrats en aluminium (Fig. 15a). Pour une sollicitation en traction-cisaillement (à $45^{\circ}$ ) une rupture par délaminage du composite (Fig. 15b) a été obtenue pour un déplacement relatif des substrats en aluminium assez faible, mais du même ordre de grandeur que celui obtenu pour un collage avec substrats en métal. La figure 15c présente une coupe d'un collage montrant les épaisseurs du composite et des deux joints de colle. Pour cet essai, il faut noter que le niveau d'effort à rupture est assez faible par la présence du composite. Comme il y a deux joints de colle pour cet essai, l'analyse du déplacement relatif des substrats en aluminium donne une valeur double des essais précédents pour un effort donné, dans la phase de comportement « linéaire ».

Des essais complémentaires doivent être réalisés pour confirmer ces premiers résultats.

\section{Influence des conditions de fabrication de la colle}

Une analyse DSC a été utilisée pour étudier la cuisson de la colle, avec des échantillons placés dans le four avec les éprouvettes « Arcan ». La figure 16 montre les résultats 


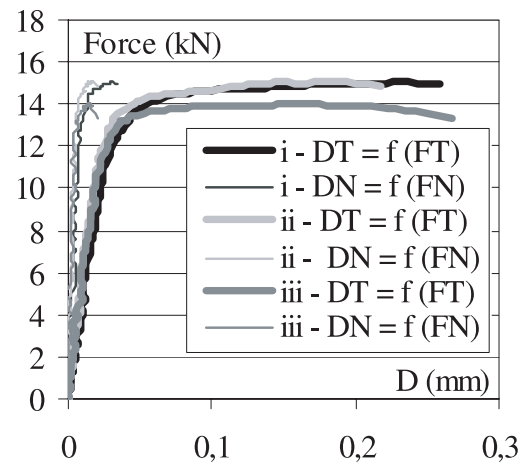

(i) aluminium - aluminium

(ii) aluminium - acier

(iii) acier - acier

(a) traction-cisaillement $\left(45^{\circ}\right)$

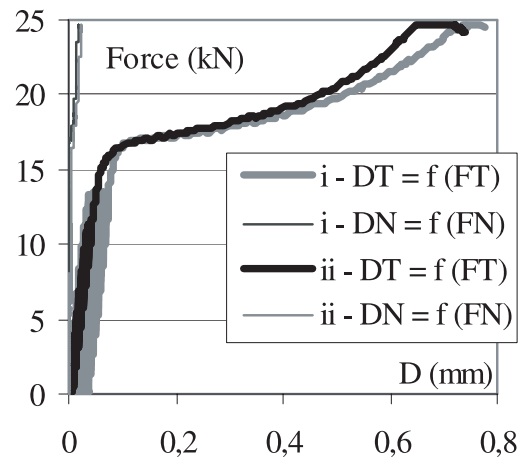

(i) acier - acier $(20$ cycles à $19 \mathrm{kN})$

(ii) alu - alu (20 cycles à $16 \mathrm{kN})$

(b) compression-cisaillement $\left(135^{\circ}\right)$

Fig. 14. Influence de la nature des substrats.

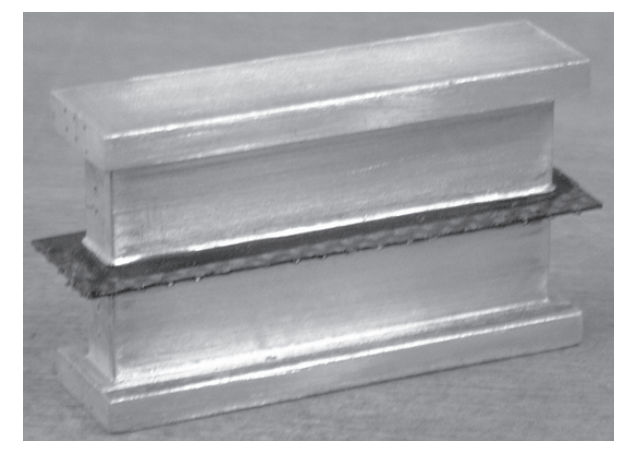

a) montage avec composite

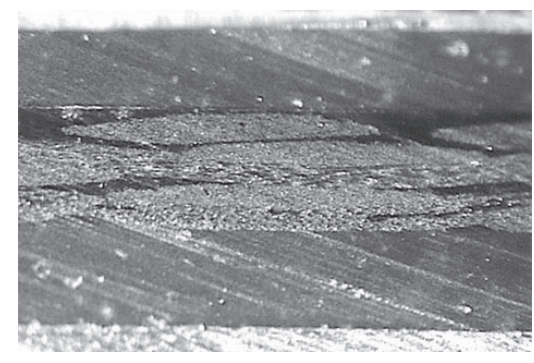

c) coupe du composite et des deux joints de colle

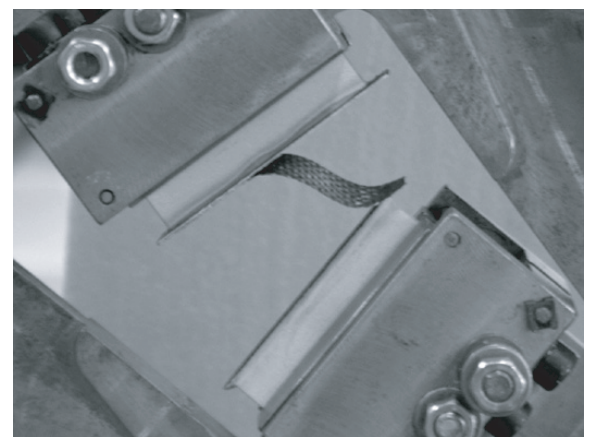

b) rupture du composite par délaminage

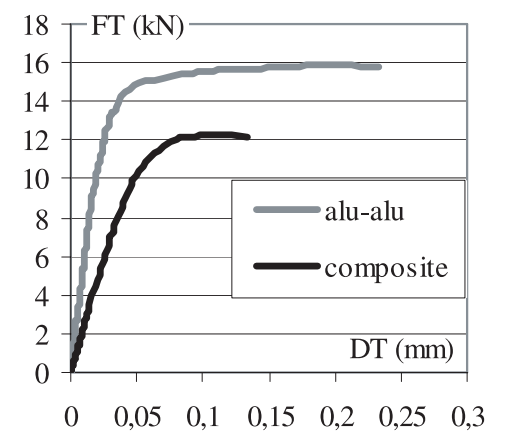

d) résultats

Fig. 15. Test de collage de composite (traction-cisaillement à $45^{\circ}$ ).

obtenus pour une analyse à $5^{\circ} \mathrm{C} \cdot \mathrm{min}^{-1}$. La température de transition vitreuse $\left(T_{\mathrm{g}}\right)$ est voisine de $40{ }^{\circ} \mathrm{C}$ et le flux de chaleur irréversible indique que la polymérisation n'a pas été complète lors de la cuisson. Une augmentation de la température de cuisson permet d'augmenter la valeur de $T_{\mathrm{g}}$, mais peut engendrer des difficultés pour un chantier naval. Cette technique a été utilisée pour vérifier que l'état de cuisson de toutes les éprouvettes était semblable; elle a l'avantage de nécessiter de faibles quantités de colle, mais elle donne peu d'information sur le comportement mécanique de la colle.
L'influence du cycle de cuisson sur le comportement mécanique en dynamique sous sollicitation de traction a été examinée pour une éprouvette de section $10 \mathrm{~mm} \times 1 \mathrm{~mm}$ (analyse DMA à $1 \mathrm{~Hz}$ ). Ces essais permettent de déterminer le module élastique $« E^{\prime} »$, le module de perte « $E^{\prime \prime} »$, pour des faibles amplitudes de déplacement imposé. La figure 16 présente les résultats obtenus pour un échantillon soumis au cycle de cuisson employé pour les essais $\left(12\right.$ heures à $20{ }^{\circ} \mathrm{C}$ suivi à 4 heures à $50{ }^{\circ} \mathrm{C}$, noté « avec $\left.\mathrm{PC} »\right)$ et pour un échantillon sans post-cuisson (noté «sans $\mathrm{PC} »$ ). Un comportement 

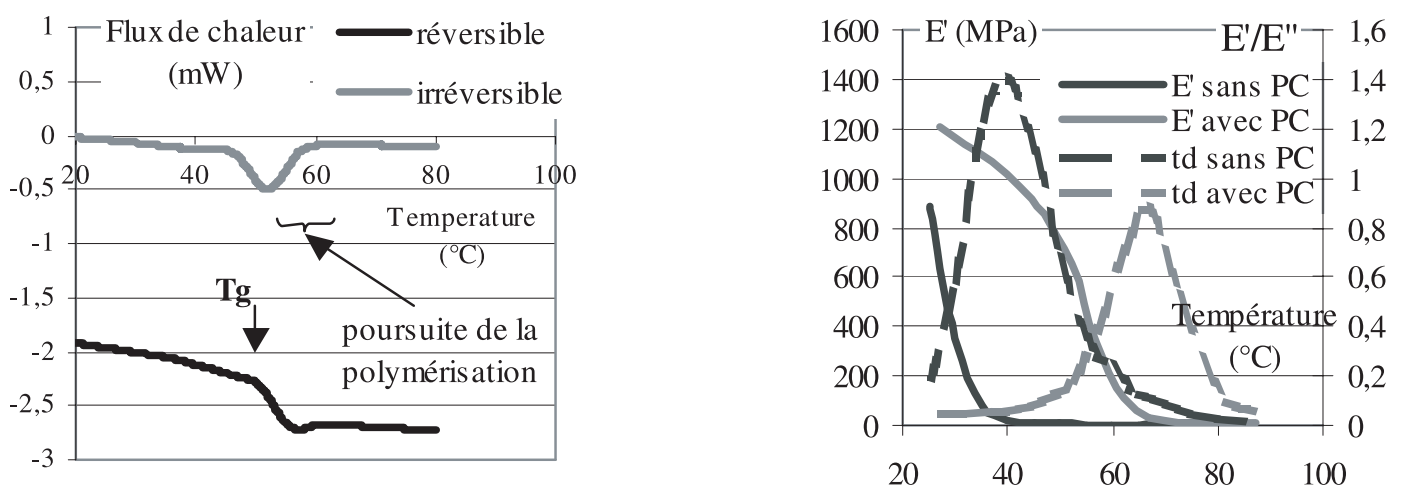

Fig. 16. Exemple d'analyse DSC et DMA de la colle.

fortement visco-élastique est observé à température ambiante, associé à une faible température de transition vitreuse, $T_{\mathrm{g}}$ (déterminé par la valeur maximale du rapport $\left.E^{\prime} / E^{\prime \prime}\right)$. Ces résultats soulignent la nécessité de prendre en compte les effets de type visqueux dans les modélisations et de bien contrôler la procédure de cuisson.

\section{Conclusions}

L'objectif à moyen terme de cette étude est de développer des outils capables de prédire le comportement de structures marines assemblées par collage. La détermination du comportement non-linéaire et des critères de rupture des composants de telles structures (composites, adhésifs) est le point de départ de ce travail. Le développement d'un dispositif de type « Arcan », avec des « becs » permettant de limiter les effets de bord, couplé avec un système de mesure sans contact permet une analyse fiable de la cinématique de la déformation du joint de la colle dans un assemblage. Cette étude a permis de déterminer l'enveloppe de rupture de la colle dans le plan contrainte normale - contrainte tangentielle. Il a été montré que la compression augmente fortement la résistance au cisaillement, ce qui est très important pour les applications sous-marines. De plus une adaptation du montage a été proposée pour une première étude du collage de composites. Les premiers résultats obtenus pour les collages mixtes (acier, aluminium, composite) montrent des comportements similaires de la colle pour la procédure proposée.

Cette étude doit être poursuivie pour développer un modèle de comportement non-linéaire de la colle, prenant en compte les aspects visqueux qui sont importants. Comme un état de déformation homogène est observé dans l'épaisseur du joint de colle, un modèle représentant l'évolution du déplacement relatif des deux extrémités du joint de colle en fonction de la contrainte appliquée peut être utilisé [18]. Sachant que la contrainte dans le plan moyen de l'adhésif, pour un comportement élastique n'est pas constante, des techniques inverses doivent être utilisées pour l'identification des paramètres du modèle.

L'influence des différents paramètres de mise en œuvre de la procédure de collage (cycle de cuisson, épaisseur du joint de colle... ) doit être étudiée pour pouvoir optimiser la fabrication des assemblages collés en fonction des sollicitations de la liaison. De plus, pour des applications marines le comportement des assemblages collés sous chargements dynamiques doit également être analysé.

Remerciements. Les auteurs remercient la Région Bretagne pour financer ce travail via un PRIR.

\section{Références}

[1] R.D. Adams, J. Comyn, W.C. Wake, Structural adhesives joints in engineering, Chapman \& Hall, 1997

[2] F.E. Penado, Analysis of singular regions in bonded joints, Int. J. Fracture 105 (2000) 1-25

[3] P. Davies, J.P. Sargent, Fracture mechanics tests to characterize bonded glass/epoxy composites : application to strength prediction in structural assemblies, in Proc. 3rd ESIS, 2003

[4] L. Tong, G.P. Steven, Analysis and design of structural bonded joints, Kluwer, 1999

[5] American Society for Testing and Materials, ASTM D5656-95, Standard Test Method for Thick-Adherend Metal Lab-Shear Joints for Determination of the StressStrain Behavior of Adhesives in Shear by Tension Loading, Annual Book of ASTM (1995) 473-478

[6] Vantico Ldt, Aerospace - Adhesive and Syntactics, Publication $\mathrm{n}^{\circ}$ A307c-GB

[7] L. Sohier, B. Gineste, P. Davies, J.Y. Cognard, 2003, Conception et évaluation d'assemblages collés de composites, in $13^{\mathrm{e}}$ Journées Nationales sur les Composites, pp. $215-227$

[8] J.Y. Cognard, P. Davies, B. Gineste, L. Sohier, Development of an improved adhesive test method for composite assembly design, Composite Science and Technology 65 (2005) 359-368

[9] M. Fischer, M. Pasquier, 1989, Shear behaviour of structural adhesives in the bondline, Construction \& Building Materials 3 (1989) 31-34

[10] L. Arcan, M. Arcan, I. Daniel, SEM fractography of pure and mixed mode interlaminar fracture in graphite/epoxy composites, ASTM Special Tech. Publ. 948 (1987) $41-67$ 
[11] D. Leguillon, E. Sanchez-Palancia, Computation of singular solutions in elliptic problems and elasticity, Masson, Paris, 1987

[12] N. Eon, Mécanismes de rupture de l'interface implantciment des tiges fémorales, thèse de l'université de Nantes, 1998

[13] M. Cheikh, P. Coorevits, A. Loredo, Modelling the stress vector continuity at the interface of bonded joints, Int. J. Adhesion \& Adhesives 22 (2001) 249-257

[14] J.P. Goncalves, M.F. Moura, P.M. De Castro, 2002, A three-dimensional finite element model for stress analysis of adhesive joints, Int. J. Adhesion et Adhesives 22 (2002) 357-365
[15] J.Y. Cognard, F. Thomas, P. Verpeaux, An integrated approach to solving mechanical problems on parallel computers, Advances in Engineering Software 31 (2000) 885-899

[16] Correlated Solutions Inc., Vic2D/Vic3D software, http://correlatedsolutions.com, 2002

[17] D. Claire, S. Roux, F. Hild, Identification de conductivités thermiques et de propriétés élastiques locales par analyse de champs, Mécanique \& Industries 4 (2003) 655-665

[18] O. Allix, A. Corigliano, Geometrical and interfacial nonlinearities in the analysis of delamination in composites, Int. J. Solids and Structures 36 (1999) 2189-2216 